\title{
Justices seigneuriales et police des communautés rurales en Anjou au XVIII ${ }^{\mathrm{e}}$ siècle
}

\section{Brigitte Maillard}

\section{OpenEdition Journals}

Édition électronique

URL : http://journals.openedition.org/abpo/1946

DOI : $10.4000 / a b p o .1946$

ISSN : 2108-6443

\section{Éditeur}

Presses universitaires de Rennes

\section{Édition imprimée}

Date de publication : 10 avril 2011

Pagination : 143-165

ISBN : 978-2-75351410-2

ISSN : 0399-0826

Référence électronique

Brigitte Maillard, « Justices seigneuriales et police des communautés rurales en Anjou au XVIII

siècle », Annales de Bretagne et des Pays de l'Ouest [En ligne], 118-1 | 2011, mis en ligne le 10 avril 2013, consulté le 30 avril 2019. URL : http://journals.openedition.org/abpo/1946 ; DOI : 10.4000/abpo.1946 


\title{
Justices seigneuriales et police des communautés rurales en Anjou au XVIII ${ }^{\mathrm{e}}$ siècle
}

\author{
Brigitte MAILLARD \\ Professeur émérite d'histoire moderne \\ université de Tours
}

La faiblesse de l'encadrement administratif et judiciaire des communautés d'habitants dans les campagnes françaises sous la monarchie absolue est patente et cette réalité reste encore avérée au $x \mathrm{XIII}^{\mathrm{e}}$ siècle, qui apparaît pourtant souvent comme le siècle de la " monarchie administrative ${ }^{1}$ ». Même les provinces qui, à l'instar de celles qui composent la généralité de Tours, Anjou, Maine et Touraine, sont proches de la capitale et font depuis longtemps partie du domaine royal et du ressort du parlement de Paris, n'échappent pas à cette règle. Les officiers royaux ne vivent pas dans les campagnes et, pour la plupart d'entre eux, ne vont pas visiter leurs administrés $^{2}$; le receveur des tailles ou de la gabelle travaille en ville et y reçoit les collecteurs villageois venus apporter l'argent de l'impôt; les juges des tribunaux royaux ordinaires, bailliages/sénéchaussées, ou spécialisés comme le grenier à sel, siègent en ville; là vit aussi le subdélégué de l'intendant. Seuls les notaires royaux, officiers ministériels, sont nombreux à être " en résidence » dans les campagnes mais ils ne sont pas établis partout. Le curé est l'unique représentant de l'autorité effectivement présent dans chaque communauté; il assume, en dehors de ses fonctions religieuses et morales, le rôle d'agent de "l'état civil ancien » mais aussi, de plus en plus souvent, celui de correspondant-informateur de l'intendant de la généralité. Les communautés s'auto-administrent sous le contrôle de ce commissaire du roi et de son représentant, le subdélégué qui réside dans une ville proche (soit pour I'Anjou, Saumur, Château-Gontier, La Flèche, Montreuil-

1. Ce texte reprend et développe la communication présentée lors des Journées régionales d'histoire de la justice, qui se sont tenues à Angers les 23 et 24 novembre 2007.

2. À l'exception des élus qui font des tournées dans leur circonscription, avant que ne soit fait le département de la taille. 
Bellay ${ }^{3}$ ). En l'absence de municipalité (jusqu'à la réforme de 1787), c'est l'assemblée des chefs de feu ou " général des habitants » qui, au cours de ses quelques réunions annuelles, prend différentes décisions, dont en particulier en Anjou, toutes celles qui concernent la levée de la taille et de la gabelle ou la gestion des communaux; le syndic, son mandataire élu, n'a aucun pouvoir de décision.

Dans la réalité quotidienne, les campagnes ne sont cependant pas un désert administratif car il y existe réellement des institutions de justice et de police, celles de la seigneurie. La géographie seigneuriale est beaucoup plus confuse que celle des communautés et paroisses : les limites du finage ne correspondent jamais avec celles d'une seigneurie, quels que soient sa taille et son rang; cependant, comme le prouvent les " actes d'éventillement ", les ruraux savent bien de quelle seigneurie les terres relèvent, et la confusion est moins grande qu'il n'est parfois dit. II n'est pas toujours facile de prendre conscience de la présence des institutions seigneuriales et de mesurer l'ampleur de leur rôle, d'autant que les situations réelles sont très diverses. Les justices seigneuriales ont longtemps été décriées mais les études récentes ont montré que, malgré leurs défauts, il n'était pas possible de les condamner totalement ou de minorer partout le rôle qu'elles jouent dans les campagnes. Elles ne peuvent pas non plus être réduites au simple rôle d'instrument du seigneur au service de la "féodalité ", même si elles constituent pour lui un moyen de faire respecter ses droits.

Quels sont les services dont une société locale a besoin dans la vie quotidienne? Ceux d'une " bonne police ». Sous l'Ancien Régime, « justice et police vont de pair », le terme de police étant à prendre dans son acception large, soit selon la définition qu'en donne Claude de Ferrrières « règlement, gouvernement et bon ordre d'une cité ${ }^{4}$ ». Dans les villes, depuis la fin du xvII siècle (1698), la police est assurée par un lieutenant général de police établi à l'instar de celui de Paris créé trente ans plus tôt (1667). Les institutions seigneuriales, souvent peu actives dans le cadre urbain, restent bien vivantes dans les campagnes. Pour en mesurer la force, l'exemple des seigneuries en Anjou est intéressant à étudier car la province semble être représentative d'une situation moyenne, entre absence et virulence. Comme l'écrit a désormais pleinement valeur de preuve juridique et que les rouages de l'administration se sont perfectionnés, le fonctionnement ordinaire de la justice produit une grande masse de papiers, de registres ${ }^{5}$ et de dossiers ${ }^{6}$. Pour l'Anjou du

3. Au XVIII ${ }^{\mathrm{e}}$ siècle, malgré des attaches avec I'Anjou et/ou le Poitou, les élections de Loudun et de Richelieu sont considérées comme relevant de la Touraine.

4. FerRiÈres, Claude de, Dictionnaire de droit et de pratique, Paris, 1772 (nouvelle édition), t. 2, p. 366.

5. En particulier, le greffier doit tenir un « registre plumitif pour servir à l'enregistrement des ordonnances, sentences et règlements qui seront rendus en la police de la juridiction de [...] pendant la présente année [...] ».

6. Voir un exemple de la diversité et de la multiplicité de ces sources dans MAUCLAIR, Fabrice, La Justice au village. Justice seigneuriale et société rurale dans le duché-pairie de La Vallière (1667-1790), Rennes, PUR, 2008, p. 341-342. 
XVIII siècle, les archives des justices des seigneurs ecclésiastiques sont assez bien conservées, en particulier celles de la baronnie de Chalonnes détenue par l'évêque d'Angers et dans une moindre mesure celles des grands chapitres; par contre pour les seigneuries laïques, y compris les grandes terres (exception faite du duché-pairie de la Vallière ${ }^{7}$ ), les fonds sont moins importants mais ils sont nombreux et permettent de brosser un tableau général des activités des officiers de la justice seigneuriale ${ }^{8}$. Ce qu'on y lit montre que les tâches de police relèvent de quelques domaines comme la surveillance de la vie économique, la police du terroir, le maintien de l'ordre...

\section{Pouvoirs et devoirs de police des justices seigneuriales}

Les principes

Dans les campagnes d'Anjou, "fief et justice sont tout un ", ainsi le seigneur a des droits de justice et de police. Le pouvoir de police est réservé aux seigneurs haut-justiciers, donc en particulier à tous les seigneurs châtelains; il découle en partie du pouvoir de ban : la coutume d'Anjou, dont la première partie s'intitule " des justices », précise dans l'article 45 que " ledit seigneur châtelain peut faire bans, édits, cris et proclamations, mettre et indire peine sur ses sujets, selon la qualité et nécessité du cas », il peut faire tenir juridiction, il peut avoir foire et marché.

Pour prendre conscience de l'étendue des tâches qui relèvent de la police seigneuriale, il suffit de consulter le traité rédigé par La Poix de Fréminville, dont le titre exact est Dictionnaire ou traité de la police générale des villes, bourgs, paroisses et seigneuries de la campagne, dans lequel on trouvera tout ce qu'il est nécessaire de savoir et de pratiquer en cette partie par un procureur fiscal dans toute l'étendue de sa justice; c'est un véritable manuel destiné tout particulièrement aux juges et procureurs fiscaux des seigneuries; l'auteur y expose, dans des rubriques classées par ordre alphabétique, les devoirs des officiers seigneuriaux et cite abondamment la jurisprudence. Ce traité a connu de nombreuses éditions, la première datant de 1756, preuves de sa large diffusion et de son utilité; il est plus simple et moins encombrant que le volumineux traité de Delamare, plutôt destiné aux officiers de police dans les villes.

7. Ibidem. Château-la-Vallière fait partie du département $d^{\prime}$ Indre-et-Loire mais avant la Révolution le duché-pairie de la Vallière était en Anjou (diocèse d'Angers, détroit de la coutume d'Anjou) bien que ressortissant au bailliage de Tours.

8. L'étude s'appuie sur le dépouillement des principaux fonds conservés (voir aux Archives départementales de Maine-et-Loire le répertoire dactylographié des justices seigneuriales laïques; les inventaires-sommaires imprimés de la série G (justice de la baronnie de Chalonnes, dont l'évêque d'Angers était seigneur), de la série H (pour la justice des chapitres), de la série E et sous-série 1E (pour la justice d'un certain nombre de seigneuries laïques). 
Les agents de la justice seigneuriale

Pour exercer la justice le seigneur doit nommer des officiers, dont la charge n'est pas toujours vénale. Les deux principaux sont le juge, appelé souvent sénéchal ou prévôt, et le "procureur fiscal ", qui sont assistés d'huissiers, de sergents, de procureurs, parfois même de commissaires de police; le greffier, dont le travail se révèle très important, est le plus souvent fermier du greffe. Le juge et le procureur fiscal sont fréquemment choisis parmi les officiers royaux de judicature en fonction dans la ville la plus proche ou portent le titre d'avocat en parlement ${ }^{9}$; beaucoup sont titulaires $d^{\prime}$ 'une licence en droit et d'autres sont notaires. Ils sont reçus après une enquête de «bonne vie et mœurs ».

La Poix définit ainsi le rôle du procureur fiscal : "Le procureur fiscal d'une seigneurie, grande ou petite, [est] chargé par état de cette partie de la Justice la plus essentielle pour la société, maintenir la religion, faire fleurir le commerce, et enrichir les sujets du roi. "Selon C. de Ferrières, il doit "défendre et soutenir les droits [du seigneur] et ceux du public ", " on lui communique les affaires où le public est intéressé [...] de manière qu'on peut dire que ce qui est prescrit par la procédure, la discipline, la police, aux procureurs du roi, convient aux procureurs fiscaux ». II dénonce les délits qui relèvent de la police, la non-exécution des ordonnances du juge, il porte à la connaissance de ce dernier les désordres existants ou susceptibles de se produire. Il a des pouvoirs propres : il peut mettre en mouvement de lui-même les instances de police sans être saisi d'une plainte; il porte plainte, il donne des réquisitions, il peut présenter au juge des remontrances afin que soit prise une ordonnance sur tel ou tel sujet. Sur requête du procureur fiscal, le juge édicte des règlements où il rappelle les règles établies dans les ordonnances de police ${ }^{10}$; il intervient soit par des textes brefs portant sur un point soit par des règlements plus complexes qui abordent différents sujets ${ }^{11}$. II peut faire des visites pour en contrôler l'application, établir les contraventions et punir les coupables. Toutefois le droit de faire des ordonnances générales n'appartient qu'au roi et à ses officiers; les textes qu'émet un juge seigneurial en permettent l'application grâce à une adaptation à la situation locale. Sur réquisition du procureur le juge fait assigner à comparaître les délinquants, les interroge au cours de l'audience et émet une sentence. Les moyens d'information de la population peuvent sembler dérisoires mais se révèlent efficaces : sans doute le juge n'a-t-il pas de mal à faire connaître ses décisions, ordonnances et règle-

9. Arch. dép. de Maine-et-Loire, E693. Dans le marquisat de la Grande Guerche, le comte de la Galisonnière fait rétablir la justice en 1776 et le juge choisi est J.-B. Louis de La Réveillère, licencié en droit, avocat en parlement, "seul juge civil, criminel et de police de lad. Juridiction ».

10. LemerCIeR, Pierre, Les Justices seigneuriales dans la région parisienne de 1580 à 1789 , Paris, Domat-Monchrestien, 1933, p. 155.

11. MauClaIR, Fabrice, La Justice au village..., op. cit., p. 301. À Château-la-Vallière, entre 1696 et 1790, 123 ordonnances de police ont été élaborées. 
ments, par voie d'affichage ou de proclamation faite par un sergent ou par le curé. Les moyens de répression sont l'amende et parfois la confiscation. Comme tous les autres le juge seigneurial agit " au nom du roi » puisque toute justice émane du roi. Les tâches sont donc bien réparties entre juge et procureur fiscal.

Les règles juridiques de la vie en commun sont d'origines diverses : textes normatifs comme les règlements seigneuriaux existants, les décisions des assemblées générales de la communauté, les arrêts du parlement de Paris, les textes royaux (ordonnances, édits et déclarations), les ordonnances de l'intendant, mais aussi la coutume ainsi que les traditions et usages locaux non écrits. C'est un ensemble souple, susceptible d'évoluer selon les besoins. Les officiers seigneuriaux en sont instruits et les citent; ils doivent d'ailleurs enregistrer les textes émanés du roi et du parlement et les faire connaître. En 1786 le procureur de Candé ${ }^{12}$ se réfère à de très nombreux textes et remonte même aux " ordonnances de saint Louis » en 1270 et à une charte de 1247. En juin 1763 à Blaison ${ }^{13}$, en "la chambre du conseil du palais ordinaire de la baronnie de Blaison, vicomté de Chemellier et autres terres ", le sénéchal arrête un règlement de police considérant qu'il « est intéressant de donner par un règlement utile, une forme certaine au bon ordre et à la police qui doit être tenue en ce bourg particulièrement et dans toute l'étendue de cette paroisse » et il fait référence aux arrêts de règlement du parlement des 4 et 10 février 1724 . À Rochefort ${ }^{14}$, le procureur remontre que " journellement on contrevient aux droits de la dame de cette cour, aux édits, arrêts et déclarations du roi, aux ordonnances et sentences de la sénéchaussée d'Angers et à celles rendues à ce siège »; il établit donc une hiérarchie entre les décisions des différentes autorités.

\section{Les justices seigneuriales en Anjou à la fin de l'Ancien Régime}

Un rapport ${ }^{15}$ établi en floréal an XIII (mai 1804), sans doute un peu passéiste et qu'il ne faut pas prendre au pied de la lettre, rend en quelque sorte hommage à ces justices locales : "Dans l'ancien ordre des choses, les tribunaux étaient très multipliés, très rapprochés dans cette province ; et tous étaient en activité. La différence quant à leur occupation n'était que sur les plus ou moins d'affaires dont chacun d'eux était ordinairement chargé [...]. » Ces justices étaient nombreuses. On en comptait vingt-sept dans le ressort de la sénéchaussée d'Angers (365 paroisses), quatorze dans celui de la sénéchaussée de Saumur (195 paroisses) et dix neuf dans celui de la sénéchaussée de Baugé (96 paroisses), en tout soixante environ, non compris l'arrondissement particulier de la juridiction royale du comté de Beaufort (10 paroisses).

12. Arch. dép. de Maine-et-Loire, 12B119; $12 \mathrm{~B} 20$.

13. Arch. dép. de Maine-et-Loire, $12 B 32$.

14. Arch. dép. de Maine-et-Loire, E693.

15. Bib. mun. d'Angers, ms $1122, f^{\circ} 178-180$. 
Il se portait et se jugeait beaucoup d'affaires dans celles de ces justices seigneuriales les plus marquantes, et qui étaient en pleine activité. Outre les matières ordinaires, civiles et criminelles, elles avaient dans leurs attributions la police, la voirie, la gruerie pour les eaux et forêts etc.

Elles avaient aussi leurs notaires particuliers créés par les seigneurs, et connus sous le titre de notaires de cour; "ils étaient très multipliés ", ce qui constituait un vrai service de proximité. Ce rapport insiste donc sur le caractère de proximité des justices seigneuriales en Anjou, sur leur densité et sur la diversité de leur champ d'action.

\section{Assurer la police économique}

Sous l'influence de la physiocratie et du libéralisme, la législation royale en matière économique change profondément au cours de la seconde moitié du XVIII ${ }^{\mathrm{e}}$ siècle. De plus les problèmes de subsistances semblent plus angoissants pour la population, alors que la production agricole connaît moins de crises dramatiques qu'au siècle précédent. II faut « réformer les abus qui se peuvent commettre dans le commerce » mais la surveillance du commerce des denrées alimentaires est l'activité la plus prenante.

\section{Surveillance des marchés}

"Celui qui a droit de châtellenie est fondé d'avoir [...] foires, marchés, sceaux de contrats, mesures à blé et à vin, dont il prend le patron à soi même » (coutume d'Anjou, art. 43). Les moyens justiciers ont aussi le droit de « bailler mesures à blé et à vin » (coutume d'Anjou, art. 40).

Il est nécessaire que les marchés se déroulent dans l'ordre; ils sont d'abord au service des particuliers qui doivent pouvoir s'approvisionner librement et convenablement sans subir la concurrence des marchands; le vieux principe de la nécessaire protection des acheteurs n'est pas abandonné. Les marchés sont encadrés par des règlements très précis. Des créations ont encore lieu au XVIII ${ }^{e}$ siècle ${ }^{16}$, toujours par lettres patentes du roi alors que les règles de fonctionnement sont édictées par le juge seigneurial. Ces règlements se retrouvent dans les archives de Chalonnes et Morannes (où les marchés sont créés en juillet 1702), Blaison (créé en 1762). Comme

16. Arch. dép. de Maine-et-Loire, G54, lettres patentes de juillet 1702 créant deux marchés sur la demande de Mgr Le Pelletier, évêque d'Angers. Le marché de Chalonnes doit se tenir le mardi, parce qu'il ne s'en tient pas ce jour-là à moins de 5 ou 6 lieues à la ronde ; celui de Morannes est fixé au jeudi. En même temps, à Chalonnes, est établi un marché aux bestiaux le premier mardi de chaque mois. Le 14 mars 1771, dans une lettre au contrôleur général Terray (Arch. dép. d'Indre-et-Loire, C95), l'intendant Du Cluzel indique que tout seigneur ayant "foire ou marché établi d'ancienneté » peut former une opposition à l'établissement d'un nouveau marché ou foire dans une étendue de 4 lieues de sa terre; il affirme aussi que comme le seigneur fournit les officiers pour veiller à la police du marché, il est juste de lui conserver les revenus du marché (loyer des bancs et des étaux...). Arch. dép. de Maine-et-Loire, 12B32 : création du marché de Blaison. 
un certain laisser-aller se fait jour avec le temps, les prescriptions sont souvent rappelées soit par des ordonnances seigneuriales, soit par des procès-verbaux d'abus qui font référence aux règlements royaux et seigneuriaux (Rochefort, la Grande Guerche en 1780, Candé en 1754). Cette abondance de prescriptions, retrouvées dans des archives qui ne sont pas complètes, montre bien l'importance attachée au maintien du bon ordre dans les marchés.

Les horaires et les lieux de fonctionnement sont déterminés de façon précise. L'heure d'ouverture varie selon la saison : à Rochefort elle est à 5 heures du matin l'été ( $1^{\mathrm{er}}$ avril-1 ${ }^{\mathrm{er}}$ octobre), 7 heures I'hiver, sans doute pour que les transactions se déroulent à la lumière du jour et que les vendeurs ne puissent pas tirer profit de l'obscurité pour tromper les chalands. Il est aussi interdit aux acheteurs de commercer plus tôt en allant au devant des marchands et de détourner ainsi des denrées du marché ${ }^{17}$. II est aussi interdit de vendre à l'intérieur des maisons. Les particuliers doivent être servis les premiers : à Rochefort, les marchands ne peuvent se mettre à acheter qu'une heure après l'ouverture du marché (donc à 6 heures ou 8 heures selon la saison). La marchandise ne peut pas être étalée n'importe où ; le règlement du marché de Chalonnes est très précis à ce sujet. Le juge doit aussi s'assurer que les règles du commerce sont respectées pour toutes les marchandises vendues sur le marché, en particulier de la part de marchands ambulants qui débitent des produits très variés, en abusant souvent de la crédulité des acheteurs, mais aussi dans les boutiques.

\section{La police des subsistances et des prix}

La police des blés peut sembler superflue au XVIII ${ }^{\mathrm{e}}$ siècle dans les campagnes censées vivre en autoconsommation et être moins dépendantes des marchés que les villes. En réalité les ruraux, dont les paysans eux-mêmes, manquent souvent de grains et en achètent de plus en plus au cours du siècle. Ils sont donc attentifs à l'approvisionnement des marchés ruraux et à l'évolution du prix, en particulier celui du seigle, alors que les citadins sont plus sensibles aux fluctuations des cours du froment.

«Comme le pain est le premier aliment et la principale nourriture de I'homme, la police ne peut avoir trop d'attention à en fixer le prix qui doit être relatif à celui des grains et à ce que les boulangers n'en vendent que de bonne qualité, bien conditionné et de poids juste » et elle doit être sensible " aux cris que font les pauvres et même les personnes aisées ${ }^{18}$ ». Les officiers seigneuriaux ont à faire respecter les règles du commerce du grain telles qu'elles sont fixées par le gouvernement et enregistrées par le parlement, même s'ils sont en désaccord; ils peuvent aussi en édicter afin d'assurer localement un bon approvisionnement et un «bon prix » pour le grain et le pain; mais il est difficile de déterminer quel doit être un «bon

17. Arch. dép. de Maine-et-Loire, 12B460.

18. Arch. dép. de Maine-et-Loire, 12B394; 12 avril 1787. 
prix ». Le cours du grain est toujours libre alors que celui du pain ne l'est pas puisqu'il est fixé par rapport à celui du blé : c'est la «taxe » du pain. Des «prud'hommes » ou jurés sont désignés par le juge seigneurial; leur fonction est d'aller sur le marché relever les prix des grains puis de les déclarer au greffier qui les inscrit sur un cahier destiné à conserver ces relevés. Cette obligation est plus ou moins bien respectée et il est rare de retrouver ces mercuriales des campagnes angevines. Elles existent à Candé ${ }^{19}$ mais seulement pour 1754 ; à Blaison, un registre (sous la forme d'un petit cahier) est commencé en 1764 mais il n'a servi qu'une fois ${ }^{20}$. Ces relevés de prix existent pourtant bien car les ordonnances seigneuriales fixant le prix du pain sont abondantes dans les archives; elles ne semblent toutefois pas être rendues à chaque marché, mais seulement à l'occasion des fluctuations du prix du grain (à la hausse ou à la baisse) : le procureur constate la variation et la fait connaître au juge qui établit alors le nouveau prix du pain; parfois la demande vient des boulangers eux-mêmes ${ }^{21}$. De plus en plus souvent, cette " taxe » est établie sur le modèle de celle d'Angers, « ville où est le siège supérieur ».

Les boulangers sont particulièrement surveillés. II faut que les boutiques et/ou le marché soient bien approvisionnés, en bon pain, au poids exact, afin que le "public » ne soit pas trompé. La pâte doit être " bien fermentée et boulangée sans mixtion », le pain bien cuit et froid quand il est mis en vente; il faut que les balances soient "suspendues à une certaine hauteur, pour que leurs bassins ne reçoivent point de contrecoup favorable au vendeur par une adresse frauduleuse 22 ». Le juge inspecte les boutiques sur réquisition du procureur fiscal quand les plaintes sont trop nombreuses; il contrôle les pains qui doivent être de "bon poids ", être marqués avec autant de trous ou de ronds, les "rondeaux ", qu'ils pèsent de livres et porter les initiales du boulanger, qui aura par ailleurs déposé sa marque au greffe seigneurial; c'est le plus souvent le défaut de " marque » qui est constaté. En cas de manquement à la règle, les pains sont confisqués et donnés aux pauvres ou à I'hôpital, tandis que le boulanger est condamné à une amende. Parfois ce dernier se rebelle et refuse de voir ses pains saisis. Les sanctions peuvent aller jusqu'à l'interdiction d'exercer.

Le commerce de la viande est moins contrôlé et ne fait pas l'objet de relevés de prix sur le marché, mais il est encadré : un prix maximum est fixé pour les viandes de bœuf, de veau et de mouton qui sont vendues à la livre-poids; le prix en est généralement de 5 à 6 sous; parfois un prix «pour les pauvres » est déterminé. La volaille n'est pas concernée. À Blaison, un «boucher juré du seigneur de la cour pour le bourg de Blaison » est nommé et les bouchers « forains » ne peuvent vendre dans ce bourg. II existe par-

19. Arch. dép. de Maine-et-Loire, $12 \mathrm{~B} 119$.

20. Arch. dép. de Maine-et-Loire, $12 \mathrm{~B} 32$.

21. Arch. dép. de Maine-et-Loire, 12B430; demande présentée par les boulangers de Saint-Georges-sur-Loire le 25 février 1769.

22. Arch. dép. de Maine-et-Loire, E693. 
fois des statuts pour ce métier : à Longué, qui est un gros bourg de 700 à 800 feux au XVIII ${ }^{\mathrm{e}}$ siècle, ils sont édictés en 1547 puis repris en 1627 et en $1771^{23}$. Les bouchers refusent assez souvent d'appliquer la " taxe », qu'ils trouvent généralement insuffisante, et ils peuvent faire montre d'insolence envers la justice : ainsi, en 1779 à Saint-Georges, I'un d'eux déclare qu'il « se fout de la justice » et de ceux qui « la mettent »; il est frappé d'une amende de 50 livres. Chaque année, à Mardi gras, le juge seigneurial désigne un "boucher de carême » qui aura seul le droit de vendre de la viande pour les malades pendant cette période de jeûne et d'abstinence. Les modalités varient selon les seigneuries; à Chalonnes la nomination se fait à l'issue d'enchères et le profit de la vente est remis aux institutions s'occupant des pauvres : en 176560 livres, en 1766130 livres, en 1789100 livres. Les bouchers de ce bourg ont différents devoirs et privilèges : au moment du carême ils doivent remettre "à la cour » un morceau de bœuf, un de veau et un de mouton ainsi que la somme de 5 sous, et ils reçoivent une chopine de vin blanc nouveau, un petit pain valant 4 sous et cinq aiguilles à coudre ${ }^{24}$. À Candé, lorsque le juge fixe le prix du pain et de la viande, il détermine aussi les prix pour les différentes qualités de son, de suif et de chandelles (selon la qualité du suif utilisé et des mèches).

Le juge seigneurial doit contrôler les poids et mesures : boisseau, aune, poinçon. À Morannes ${ }^{25}$, le 14 septembre 1784, une inspection est menée chez les boulangers, bouchers et marchands d'étoffe; aunes et poids défectueux sont saisis et déposés au greffe du tribunal. En 1708 à Blaison, à Vernoil en $1786^{26}$, on procède à un étalonnage des boisseaux. Ce même souci de surveillance fait que les tonneliers doivent désigner des jurés, alors que les campagnes ignorent les jurandes.

Le juge doit aussi s'assurer que les tarifs des péages sont bien affichés : le 30 avril 1774 est dressé à Blaison le procès-verbal de la plantation de poteaux portant les tarifs du bac du Port-en-Vallée. Toutefois les archives dépouillées ne contiennent pas de procès-verbaux concernant le fonctionnement des péages, bacs ou ponts, qui sont pourtant nombreux dans la province.

\section{La lutte contre les troubles de subsistances}

La moindre inquiétude au sujet du pain quotidien peut se traduire par des réactions violentes, de la part des femmes en particulier. Or l'évolu-

23. Brizay, François, Follain, Antoine et Sarrazin, Véronique (dir.)., Les Justices de Village. Administration et justice locales de la fin du Moyen Âge à la Révolution, Rennes, PUR, 2002, p. 365-369 : transcription par Antoine Follain des « coutumes » et « statuts » des bouchers de Longué en 1547, 1625 et 1771.

24. Il est probable que le défilé du « bœuf gras » existe dans différents bourgs mais les archives consultées n'en gardent pas trace.

25. Arch. dép. de Maine-et-Loire, 12B394; 1784.

26. Arch. dép. de Maine-et-Loire, 12B32. Arch. dép. de Maine-et-Loire, $12 B 460$. 
tion rapide en faveur de la liberté ${ }^{27}$ pour le commerce des grains dans la seconde moitié du siècle est mal comprise par les populations. Les modifications apportées aux règles du commerce et du transport des grains ont été difficilement appliquées; elles allaient trop contre la tradition et passaient pour mettre en danger l'approvisionnement local; les populations se sentant menacées par la disette et donc la cherté, expriment à partir des années 1765 un fort ressentiment qui prend parfois la forme d'émeutes de marché ou des " arrêts de grains ${ }^{28}$ ». Les prix du grain et du pain continuent à connaître de fortes fluctuations : à Candé, en 1782, entre janvier et juin l'augmentation a été de $25 \%$ pour le meilleur pain, de $40 \%$ pour le pain de seigle, le pain des pauvres, qui a, comme lors de toutes les «crises de cherté ", subi la hausse la plus marquée; entre la mi-avril 1788 et la mi-avril 1789 le prix du pain « mollet » a crû de $52 \%$, celui du pain de méteil de $65 \%$ et celui du pain noir de $82 \%$ (il atteint 1 sou 8 deniers).

Dans ces circonstances, les autorités seigneuriales ont un rôle essentiel à jouer car elles sont les premiers témoins de la naissance et du développement des troubles. À Craon ${ }^{29}$, dont la baronnie appartient au marquis d'Armaillé, le marché, pour des raisons pratiques, n'est pas approvisionné en grains mais en farines; en 1766 le procureur fiscal, qui a le soutien du marquis, est en guerre ouverte contre les meuniers : il entend fixer les prix des farines à un niveau inférieur à celui que pratiquent ceux-ci et il surveille rigoureusement les transactions. L'intendant Du Cluzel est très embarrassé devant cette situation inédite car si la législation porte sur le commerce des grains, il n'est rien prévu pour celui des farines. Lorsque des violences se produisent, la collaboration doit être étroite entre les juges seigneuriaux et la maréchaussée, qui constitue la seule force armée pour le maintien de l'ordre dans les campagnes et qui a reçu par l'arrêt du conseil du 19 mai 1739 la connaissance " des séditions, émotions et attroupements qui pourraient survenir à l'occasion des bleds et autres grains ». C'est le cas lors des émeutes de subsistances qui se développent dans les années 1770 , et dont les plus graves n'ont pas épargné I'Anjou ${ }^{30}$. Cependant ses effectifs sont très faibles ${ }^{31}$ et, dans ces temps de grande tension, elle ne peut être présente sur tous les théâtres de troubles, qui se localisent en particulier sur les marchés et le long des cours d'eau (Mayenne, Sarthe), là où se font les expéditions de grains (Montreuil, Pruillé...). Le rôle joué par les officiers seigneuriaux dans ces circonstances n'est pas encore bien connu, alors

27. Kaplan, Steven L., Le Pain, le peuple et le Roi. La bataille du libéralisme sous Louis $\mathrm{XV}$, Paris, Perrin, 1986.

28. TILLY, L.-A., "La révolte frumentaire, forme de conflit politique en France ", Annales, Économies, Sociétés, 1972, Nº 3, p. 731-757.

29. Arch. dép. d'Indre-et-Loire, C 96.

30. Nicolas, Jean, La Rébellion française. Mouvements populaires et conscience sociale 1661-1789, Paris, Seuil, 2002, p. 221-265.

31. Arch. dép. d'Indre-et-Loire, C77. En 1780, il y a en Anjou deux lieutenances de la maréchaussée; celle d'Angers compte 9 brigades et 42 hommes y compris le prévôt général, celle de Château-Gontier 4 brigades et 39 hommes tout compris. Ainsi la brigade de Pouancé, située aux frontières de la Bretagne, compte un maréchal des logis et 3 cavaliers. 
qu'ils étaient les seuls représentants de l'ordre à être vraiment présents sur le terrain.

Le juge seigneurial a tout intérêt à ce que les travaux agricoles se déroulent sans entraves et à ce que la production agricole soit suffisante pour que les paysans puissent faire face à leurs obligations financières (paiement des impôts, de la rente foncière...) et aux nécessités de la vie quotidienne. C'est pourquoi une grande attention est portée au maintien du bon ordre dans le finage ${ }^{32}$.

\section{Assurer la police de la vie agraire}

L'Anjou ne fait pas partie des pays où les pratiques communautaires dans les campagnes sont la règle, ce qui ne veut pas dire que l'individualisme y règne en maître et qu'il n'existe aucune régulation. Le juge seigneurial est chargé de la police du finage mais quand il ne l'assure pas, les communautés prennent le relais pour sauvegarder les récoltes les plus importantes. En Anjou il intervient essentiellement dans deux domaines: la fixation du ban des vendanges et la surveillance des prés et pacages, communs ou non.

\section{Le ban des vendanges}

La fixation de la date d'ouverture pour les vendanges découle du pouvoir de ban des seigneurs (il figure dans les coutumes d'Anjou, Maine, Bourges et Bourbonnais); par exception ce droit n'est pas réservé aux châtelains : "Le droit de mette [le ban des vendanges], c'est à dire, de fixer le jour auquel doivent commencer les vendanges, n'est point une prérogative de la châtellenie ni même de la haute justice; ce droit se règle par la possession, et ceux qui l'ont pour eux y doivent être maintenus ${ }^{33}$. " La coutume d'Anjou précise que «le ban de vendanger n'est réputé prérogative de châtellenie ni haute justice, et pour ceux qui sont en possession ancienne d'en user en jouiront » (art. 185 et 186). Selon La Poix de Fréminville " dans les pays où il y a des vignes, c'est aux officiers de police de donner le ban des vendanges ". La proclamation est faite très régulièrement, chaque année, et c'est peut-être la démarche qui a laissé le plus de traces matérielles dans les archives seigneuriales (on a en particulier une longue série pour la baronnie de Blaison). La procédure est bien établie et respectée. Le but est d'empêcher les vols de raisin et de faciliter la perception de la dîme. C'est au procureur fiscal de prendre l'initiative, en répondant parfois à la demande des habitants qui jugent que la récolte sera en danger si les vendanges tardent trop : il convoque alors l'assemblée qui devra nommer

32. MauClaiR, Fabrice, La Justice au village..., op. cit., p. 299. À Château-la-Vallière, entre 1696 et 1790 , les officiers seigneuriaux ont émis 123 ordonnances de police, sur lesquelles plus d'une sur cinq concernait les récoltes.

33. Trottier, Principes des coutumes d'Anjou et du Maine, Angers, Mame, 1783, t. 1, p. 23-24. 
des experts pour inspecter la vigne. Après avoir entendu leur rapport, elle donne son avis et le juge seigneurial prend une ordonnance; il entérine le plus souvent la date choisie par l'assemblée : "ce requérant le procureur fiscal et après avoir fait l'enquête et pris l'avis des notables de cette paroisse et de celle de Chemellier en la manière ordinaire et accoutumée de la maturité des fruits de vendanges et de la nécessité d'en établir le ban et ouverture pour la présente année 1719 , nous avons assigné le ban des vignes de cette paroisse au lundi deuxième d'octobre prochain et pour l'étendue de ladite paroisse de Chemellier au quatrième jour dud. Mois ${ }^{34}$ ". II rappelle toujours l'interdiction de " grapiller » ou " grapper » avant la fin de la vendange. Fréquemment deux dates sont choisies, l'une pour les "vendanges rouges » et l'autre pour les « blanches ». Des désaccords peuvent cependant se manifester, certains jugeant la date trop tardive ou trop précoce. Quelques-uns trichent : par exemple, ceux qui n'ont pas de pressoirs ${ }^{35}$ vendangent un peu avant le ban et pensent être en règle parce qu'ils ont requis l'autorisation du décimateur. Dans quelques cas les habitants demandent à vendanger plus tôt car les conditions météorologiques sont défavorables, que le raisin pourrit.

Les dossiers étudiés ne contiennent aucun texte concernant le droit de glaner.

\section{La protection des prairies}

Dans les campagnes angevines l'élevage occupe une place qu'il ne faut pas sous-estimer, et donc l'usage des prés et des communs doit être surveillé, en particulier lors des années où le temps est déréglé. De très nombreux règlements concernant le pacage sont ainsi conservés dans les archives de la justice de la baronnie de Blaison ${ }^{36}$ qui démontrent l'attention que portaient les officiers seigneuriaux à ces problèmes, mais aussi la diversité des pratiques et celle des délits qu'ils avaient à traiter ${ }^{37}$.

La coutume d'Anjou est silencieuse sur les usages dans les prairies; les vignes sont " défensables par nature » mais non les pâturages qui sont souvent, en particulier dans la vallée de la Loire, constitués en partie de communs. Les vignes doivent être encloses et lorsque les propriétaires ou locataires négligent de veiller à ce qu'elles le soient, les animaux y pâturent "au mépris des ordonnances », alors qu'il est défendu d'y laisser "vaguer des bestiaux » ou d'y couper de l'herbe ${ }^{38}$. Le seigneur a un

34. Arch. dép. de Maine-et-Loire, $12 \mathrm{~B} 32$.

35. II n'existe pas de banalité du pressoir en Anjou.

36. Arch. dép. de Maine-et-Loire, $12 \mathrm{~B} 32$.

37. Follain, Antoine, et Lemoine Estelle, "Réguler par soi-même ou s'en remettre aux juges? Des communautés et juridictions d'Ancien Régime aux municipalités et administrations de la France contemporaine », dans : Follain, Antoine (dir.), Les Justices locales dans les villes et villages du Xve au xIXe siècle, Rennes, PUR, 2006, p. 56-67.

38. Arch. dép. de Maine-et-Loire, 12B424, ordonnance du 15 septembre 1769 (abbaye de Saint-Florent de Saumur). 
droit de contrôle sur les communaux si ceux-ci ont été concédés à cens. La tradition veut que les prés soient "défensables » du début de mars jusqu'à l'enlèvement de la première herbe. Sur demande de l'assemblée de communauté, dont l'avis devient le plus souvent la norme qu'il retient, le juge seigneurial peut prendre des ordonnances limitant le nombre de bêtes admises sur les prairies communes pour les réserver au gros bétail, ou interdire le pacage des moutons qui arrachent l'herbe et/ou des oies qui « l'empestifèrent ${ }^{39}$ ». Le 2 octobre 1769, le sénéchal de Bécon et du Plessis-Macé rend une ordonnance concernant les droits d'usage sur les landes en application du titre 19 de l'ordonnance de 1669 : tous ceux qui ont des droits sur les landes doivent faire une déclaration des bêtes qu'ils entendent y mettre, à faire enregistrer au greffe; ils doivent marquer leurs bêtes et leur mettre au cou des clochettes afin qu'on puisse les retrouver si elles s'échappent; il est interdit d'y mettre des ovins et des caprins; seuls les ayants droit peuvent y mettre à pacager des bêtes leur appartenant et ils ne peuvent pas « prêter leur nom à des habitants des villes ou paroisses voisines » ni y mettre des bêtes prises en bail à cheptel. Le procureur fiscal doit faire punir les délits : ainsi il observe si des particuliers coupent I'herbe avant sa maturité, si des bêtes sont envoyées sur les prés avant qu'ils ne soient devenus " communs » et même avant que l'herbe n'en soit totalement coupée : « différents particuliers laissent vaguer leurs bestiaux avant que l'herbe en soit totalement fauchée et enlevée, que même leurs faucheurs et faneurs sont plus occupés à détourner lesdits bestiaux qu'à travailler, joint que ces bestiaux mangent et foulent l'herbe avant qu'elle soit sèche, ce qui leur porte perte de toute façon ", note-t-il à Blaison en 1737. Les mêmes abus se reproduisent en 1768 ; le 2 juillet, on y compte près de 100 bêtes "tant chevaux que vaches", les menaces d'amende et de confiscation ayant peu d'effets; une enquête est ouverte et huit témoins désignent un certain nombre de propriétaires d'animaux qui sont assignés à comparaître mais rien n'indique quelles suites furent données. Les bêtes trouvées « en contravention » peuvent être saisies et mises en fourrière ${ }^{40}$.

Le juge impose aussi des limitations aux droits de pacage lorsque, à la suite d'une crue de la Loire, l'herbe des prairies situées dans la vallée a été abîmée et ne peut être exploitée selon les règles habituelles, mais les communautés sont toujours consultées ${ }^{41}$. Lors de la grande sécheresse de 1785 on observe une collaboration poussée entre les magistrats du parlement, les officiers des justices royales et ceux des justices "subalternes » dans le but de préserver au maximum la possibilité d'avoir des regains. Les

39. Arch. dép. de Maine-et-Loire, 12B20. Règlements pour les baronnies de Bécon, le Plessis-Macé (1769) portant sur la quantité et la nature des bêtes qui peuvent profiter des droits d'usage sur les prés. De telles défenses se retrouvent dans toute la région ligérienne (Touraine, Sologne) et ailleurs (MORICEAU, Jean-Marc, Histoire et géographie de l'élevage du Moyen Âge à la Révolution, Paris, Fayard, 2005, p. 181-182).

40. Arch. dép. d'Indre-et-Loire, 33B87; baronnie de Bourgueil ; PV daté du 8 juillet 1770 pour la saisie de six bovins ayant pacagé sur des prés avant que le foin ait été enlevé.

41. Arch. dép. de Maine-et-Loire, 12B32, (juillet 1738). 
décisions sont prises sans attendre celles du gouvernement ou du parlement : la présence sur place des officiers seigneuriaux permet une réaction immédiate aux différentes situations et les mesures adoptées se révèlent plus appropriées aux conditions locales que celles décidées à Paris par le parlement, ou plus près, à Angers par le présidial, même si ses membres sont des propriétaires fonciers intéressés au premier chef par les difficultés de l'agriculture. Le 31 mai le procureur fiscal de Blaison demande, avec I'accord des habitants consultés le 29 mai, que l'ouverture des prairies qui se fait habituellement le 22 juillet soit repoussée jusqu'au 22 septembre; mais à Morannes ${ }^{42}$, lorsque l'impossibilité de voir pousser une " seconde herbe » est constatée, la prairie est ouverte aux bestiaux. À Vernoil ${ }^{43}$, une " taxe » des fourrages est décidée. L'arrêt du parlement réglementant le commerce des foins pour réserver celui-ci aux consommateurs locaux a été largement diffusé et se retrouve dans de nombreux fonds d'archives.

D'autres circonstances peuvent provoquer l'intervention du juge seigneurial dans la vie rurale. En 1765, le juge du comté de Serrant constate la prolifération des nids de chenilles dans les arbres, situation qu'il attribue à la négligence des paysans, et il ordonne leur destruction.

Le juge est souvent saisi aussi d'affaires concernant le braconnage, les coupes de bois illégales, mais selon le juriste Trottier, les droits de garenne, de chasse... dépendent plus du fief que de la justice et ne relèvent pas directement de la police.

Le maintien de l'ordre public quotidien

et la sauvegarde de la sécurité

Le juge seigneurial doit faire appliquer la législation royale dans le domaine de l'ordre public; il doit aussi obéir aux ordres émanant des officiers du roi, en tout premier des magistrats du parlement de Paris, et de I'intendant de Tours, principal représentant du souverain, même si cette exécution peut se révéler difficile. En février 1691 le sénéchal de la baronnie de Bécon fait procéder au tirage au sort d'un soldat de milice en exécution de l'ordonnance de l'intendant Hue de Miroménil en date du 23 janvier $1691^{44}$. La maréchaussée patrouillant surtout près des grandes routes et s'occupe en particulier des problèmes de vagabondage ${ }^{45}$, tous les petits désordres locaux sont traités par les officiers seigneuriaux.

42. Arch. dép. de Maine-et-Loire, $12 \mathrm{~B} 394$.

43. Arch. dép. de Maine-et-Loire, 12B460.

44. Arch. dép. de Maine-et-Loire, 12B20.

45. Les officiers seigneuriaux, souvent informés par la « rumeur publique » peuvent faire arrêter des étrangers ou des passants pour opérer une vérification de leurs papiers et procéder à un interrogatoire. 
«Empêcher le scandale public »

Au $x V I I I{ }^{e}$ siècle, le plein effet de la réforme catholique se fait sentir dans les campagnes où le ministère sacerdotal est exercé par des curés qui ont tous été formés dans un séminaire; le culte du Saint-Sacrement, le respect du dimanche, le besoin de discipline s'imposent progressivement. Curés et officiers seigneuriaux collaborent pour faire régner un ordre qui correspond à leurs conceptions de la vie religieuse et sociale ${ }^{46}$.

L'Église reçoit le soutien des autorités de justice dans sa volonté de sacralisation des dimanches et jours de fête. Le cabaret est bien l'ennemi $n^{\circ} 1$, le concurrent de l'église, et l'abus de vin est considéré comme la principale cause de désordre. La lutte des juges et procureurs fiscaux contre les cabaretiers semble aussi répétitive que vaine. Le juge fait souvent référence aux règlements généraux (comme le règlement royal du 15 novembre 1711 ou l'arrêt de règlement du parlement de Paris du 12 février 1724, qui prohibe la fréquentation des cabarets pendant l'office divin) et seigneuriaux déjà édictés et qui ne sont pas respectés. À Blaison, il est prescrit que l'interdiction soit affichée tous les trois mois à " la barre du palais » et soit publiée tous les six mois aux prônes. La réitération est éclairante sur l'attitude des autorités, des notables qui se heurtent à la désobéissance du public mais s'obstinent. Il est défendu de vendre du vin pendant les offices divins (messe et vêpres) les dimanches et jours de fêtes : à Blaison c'est entre 8 heures 30 et 10 heures du matin en toute saison et entre 2 et 3 heures de l'après-midi en hiver, de 3 à 4 heures en été. L'ordonnance portant ces interdictions doit être affichée en bonne place dans les cabarets et les auberges. Plus généralement les cabaretiers ont aussi défense de vendre du vin après 8 heures du soir entre le $1^{\text {er }}$ novembre et le $1^{\text {er }}$ avril (ou Pâques), après 10 heures du soir en été. Ces prescriptions se retrouvent partout et tout au long du siècle : ainsi à Vernoil en juillet 1730, à Blaison en juin 1763, à Bécon en novembre 1773, Candé en septembre 1780. Les juges s'emploient à ce que ces défenses ne restent pas lettre morte. À Longué ${ }^{7}$, en avril 1768, des poursuites sont engagées contre un cabaretier et ses clients trouvés en train de boire et de jouer pendant les vêpres. II est aussi interdit de tenir "boutiques ouvertes » les dimanches et jours de fêtes; les vendeurs ne doivent pas déballer et exposer leurs marchandises trop près de l'église; la distance est de 30 pieds $^{48}$ à Saint-Georges en 1780. À NotreDame-du-Pé ${ }^{49}$, le jour de "l'assemblée » du 8 septembre 1784, le juge tente sans succès d'obliger colporteurs et marchands «forains » à remballer leurs marchandises.

46. À Château-la-Vallière, les sujets concernant la religion occupent $12 \%$ de l'activité du juge seigneurial.

47. Arch. dép. de Maine-et-Loire, 12 B344.

48. Soit environ 7 mètres.

49. Arch. dép. de Maine-et-Loire, 12 B394. 
Le clergé dénonce avec énergie les désordres et débauches qui se produisent à l'occasion des processions ${ }^{50}$, qui sont un temps fort de la vie sociale et religieuse des paroisses, que ce soit aux Rogations, pour la fête du saint patron mais surtout à l'occasion de la fête-Dieu qui est « la plus solennelle » et prend de plus en plus d'importance au XVIII siècle. Les autorités seigneuriales édictent des règlements très précis : ainsi à Candé ${ }^{51}$, en 1782 , le sénéchal rappelle qu'il faut nettoyer les rues, tendre les maisons, mais aussi qu'il est défendu « de tirer des artifices ou armes à feu dans les rues, cours et jardins, ni par les fenêtres des maisons pendant que la procession du Saint-Sacrement passera dans les rues avant qu'elle passe ni même après qu'elle aura passé ». À Longué, l'assistance à la procession est obligatoire : au début de la cérémonie un sergent fait l'appel de tous les habitants afin qu'ils tiennent « leur rang et place » dans le cortège; des poursuites sont engagées contre ceux qui sont absents à l'appel, ou qui n'ont pas tenu leur place ou qui sont absents à la cérémonie; en 1772 ils encourent une amende de 20 sous. À la fin du siècle à Chalonnes, gros bourg de 884 feux en 1782, un règlement détaillé de la procession est élaboré chaque année pour lutter contre "la confusion des rangs »; il fixe le protocole à respecter pour éviter qu'on y marche sans ordonnance, ce qui provoque des troubles puisque « les notables qui portent des cierges se trouvent d'ordinaire ou confondus avec des gens vêtus indécemment qui disputent et prennent les premières places, ou arrêtés par ceux qui refusent de suivre l'alignement et l'ordre que les officiers de la baronnie ont coutume de donner aux deux rangs qu'ils forment ». Le souci de bienséance inspire les officiers seigneuriaux : le modèle à suivre est clairement urbain, c'est celui des règlements fixés par "messieurs du siège de police de la ville d'Angers notre capitale »; le cérémonial doit donc être copié sur celui des villes. L'ordre social se manifeste à la vue de tous dans les processions qui sont les principaux, et parfois uniques, défilés que connaissent les campagnes.

Les juges seigneuriaux ont aussi fait appliquer l'édit du roi de 1784 qui interdit de sonner les cloches pendant les orages et qui réglemente strictement leur usage : le 25 novembre 1784 le juge de la Grande Guerche ${ }^{52}$ publie l'acte royal et l'accompagne d'une ordonnance seigneuriale pour son application. II rappelle que l'usage des cloches n'est pas libre et est sévèrement réglementé : elles sonnent pour annoncer offices et prières; elles servent aussi à convoquer l'assemblée de communauté ou de fabrique mais une seule cloche doit alors être employée; en tout autre cas l'autorisation du curé doit être obtenue. II est depuis longtemps absolument interdit de sonner le tocsin sans l'avoir obtenue afin de conserver à la sonnerie sa signification d'urgence ${ }^{53}$.

50. Le juge est parfois aussi amené à prendre des mesures pour assurer l'ordre à l'intérieur de l'église mais il n'en reste pas de traces dans les archives consultées.

51. Arch. dép. de Maine-et-Loire, 12B119.

52. Arch. dép. de Maine-et-Loire, E694.

53. En février 1774, après les émeutes qui ont occasionné le pillage de bateaux de grains sur la Loire et le Cher, le présidial de Tours condamne à mort et fait exécuter deux vignerons pour avoir sonné le tocsin sans autorisation du curé. 
Police des rues et voirie, hygiène

Le juge seigneurial est aussi chargé de faire respecter l'ordre, la propreté et la sûreté dans les rues. II prend donc des ordonnances ${ }^{54}$ pour assurer la sécurité des passants; il ordonne d'enlever les obstacles qui pourraient s'y accumuler : il faut empêcher la présence des tas d'ordures, de bois... dans les rues et sur les ports, " ce qui cause une infection notable et empêche le passage ". II est interdit de jouer aux boules, aux quilles ou " aux bâtonnets » dans les rues et sur les places publiques ${ }^{55}$; les parents sont responsables pour leurs enfants. Les habitants doivent aussi participer aux travaux d'entretien de la voirie locale et, par exemple à Candé en mai 1733, charroyer des pavés et du sable devant chez eux " attendu que le paveur commencera à travailler » d'ici quelques jours ${ }^{56}$. II est interdit de construire sans en avoir reçu la permission; c'est le juge seigneurial qui délivre les autorisations de travaux et veille à ce que soient respectées les règles d'alignement; c'est pourquoi il fait faire des procès-verbaux d'alignement avant d'accorder le permis. Jusqu'au milieu du xvIII siècle, les seigneurs semblent peu s'occuper de la voirie; ensuite leur attitude change et les conflits avec les trésoriers des finances du bureau de Tours ${ }^{57}$ deviennent fréquents, mais le parlement prend systématiquement le parti des seigneurs : dès 1720 il maintient le duc de la Trémoïlle dans ses droits de voirie à Candé aux dépens des officiers du roi; cet arrêt a fait jurisprudence.

\section{Prévention et assistance}

Une mesure d'ordre et de sécurité est souvent édictée : elle vise la lutte contre les chiens errants, souci récurrent parce qu'on les soupçonne d'être enragés. Le 16 avril 1782, le juge de Chalonnes rappelle aux propriétaires de chiens qu'il est interdit de les laisser vaguer, qu'il faut les « tenir à la chaîne ou à la corde dans les endroits où ils ne pourroient les détenir autrement » et que, faute de quoi, les passants sont autorisés à tuer ces bêtes ${ }^{58}$; à Bécon ils doivent porter au cou " une billette de la longueur de dix pouces et cinq pouces de circonférence ». Les animaux soupçonnés d'être enragés doivent être abattus immédiatement ainsi que les bêtes qu'ils auraient mordues, les corps devant être enterrés profondément dans des fosses d'où ils ne puissent pas être déterrés. En avril 1769, à Bécon, où des porcs ont été mordus par un chien soupçonné d'avoir la

54. Entre 1679 et 1790, le juge de Château-la-Vallière a traité 11 fois (sur 147) des rues et chemins dans les ordonnances et règlements de police qu'il a pris.

55. Arch. dép. de Maine-et-Loire, G56 (7 juin 1775), E693 (1760). C'est évidemment à la fin du printemps ou en été que ces interdictions sont stipulées.

56. Arch. dép. de Maine-et-Loire, 12B119.

57. CAILlou, François, Une Administration royale d'Ancien Régime : le bureau des finances de Tours, Tours, Presses universitaires F. Rabelais, 2005, t. 1, p. 484-486.

58. À Longué, en juin 1768, il est rappelé qu'il est interdit de lâcher des chiens s'ils ne sont pas munis de «landons » autour du cou, pour éviter qu'ils ne chassent. 
rage ${ }^{59}$, il est ordonné que les bêtes soient tenues enfermées pendant " un an entier depuis ce jour, sans pouvoir les laisser vaguer dans ce bourg »; de plus, elles doivent être marquées et ne peuvent être vendues. À Candé, des ordonnances sur le même sujet sont prises en 1732, 1782, 1783, 1785. À cette occasion le procureur et le juge font référence à une ordonnance de I'intendant de Champagne du 24 septembre 1764, mais aussi aux articles 12 et 14 du titre 17 de l'ordonnance de 1667. De même le juge peut ordonner une battue aux loups : le 15 avril 1714 le juge de Durtal en prescrit une pour le 24 du mois mais interdit aux paysans de venir armés, sauf à y être expressément autorisés ${ }^{60}$. En 1748, par ordre seigneurial des battues aux loups sont organisées autour de Baugé ${ }^{61}$.

L'incendie est un fléau redouté et des mesures de prévention sont parfois prises : à Château-la-Vallière, un règlement est établi en 1705 après qu'un important incendie ait ravagé le bourg le 15 avril $1700^{62}$. Le 14 avril 1775, dans la paroisse de Chemellier, le procureur fiscal ordonne que I'assemblée générale se réunisse pour constater les dégâts et pour qu'un procès-verbal soit établi après un incendie qui venait de détruire en entier le village du May qui abritait quinze familles dont les maisons étaient couvertes de "brandes, de boures ${ }^{63}$ et d'ardoises ", car il est de son devoir " de veiller pour qu'il soit pourvu au secours et au soulagement de ces malheureux incendiés ${ }^{64} »$.

Les juges seigneuriaux sont aussi chargés de faire faire la levée des cadavres trouvés dans l'espace public; ainsi le 21 décembre 1781, le juge de Morannes ${ }^{65}$ dresse procès-verbal du décès d'un homme trouvé mourant dans un chemin et transporté à I'hôpital; c'est le cocher de madame de Collasseau et on trouve sur lui deux petits sacs de cuir renfermant en tout 456 livres que sa femme, qui réside près de La Flèche, réclame quelques jours plus tard. En application du « droit d'épave » du seigneur haut-justicier, le juge doit aussi faire la levée des enfants abandonnés sur les terres

59. Arch. dép. de Maine-et-Loire, 12B20.

60. Le 15 avril 1713, sur la remontrance du procureur des eaux et forêts, le juge du comté de Durtal rend une ordonnance ordonnant des battues dans la forêt de Chambiers, les habitants devant se réunir " avec gaules et bâtons, sans aucunes armes à feu à la réserve de ceux qui seront indiqués » (texte cité dans BrizAY, François, Follain, Antoine et SarRazin, Véronique (dir.)., Les Justices de Village..., op. cit., p. 375).

61. MoricEAU, Jean-Marc, Histoire du méchant loup. 3000 attaques sur I'homme en France $x v^{e}-x x^{e}$ siècle, Paris, Fayard, 2007, p. 157. Le procureur fiscal dénonce les ravages dus aux loups. Dans cette même période, des battues aux loups sont organisées dans de nombreuses autres régions de la généralité de Tours (autour de Tours, Amboise, Loches, Chinon, Château-du-Loir, dans la forêt de Perseigne etc.). La période de la fin du XVII et au début du xvIII ${ }^{\mathrm{e}}$ siècle, correspond à une forte poussée des attaques des loups en Touraine et en Anjou.

62. Arch. dép. d'Indre-et-Loire, 7B116. MAUCLAIR, Fabrice, GIRARD, Isabelle, « Les incendies de village dans le nord de la Touraine au XVIII ${ }^{\mathrm{e}}$ siècle ", Bulletin de la Société Archéologique de Touraine, 2005, p. 215-224.

63. Sans doute de la paille.

64. Arch. dép. de Maine-et-Loire, 12B32. Le total des pertes est évalué à $29930 \mathrm{~L}$.

65. Arch. dép. de Maine-et-Loire, 12B394 (châtellenie de Morannes). 
du fief, s'occuper de les faire éventuellement baptiser et de les faire prendre en charge par une nourrice ${ }^{66}$.

Un chirurgien désirant exercer dans une communauté doit se faire recevoir par le juge seigneurial et montrer ses titres ${ }^{67}$. Sur requête du chirurgien du village, soucieux de la santé publique mais aussi de ses intérêts, le juge peut faire contrôler les marchands ambulants qui, vendant des drogues variées, passent dans les villages et abusent de la crédulité du public. Ainsi le 27 janvier 1761, le juge de la baronnie de Chalonnes interdit à un individu de vendre des " onguents " au public sans avoir le "privilège » de le faire. Les habitants trouvent aussi sur place différentes pommades et drogues : le 11 mai 1750, à la demande du chirurgien du village qui en a le monopole, le juge de Morannes ordonne que soient contrôlées les drogues mises en vente dans les boutiques; la présence d'arsenic chez les épiciers n'est pas rare $^{68}$.

Néanmoins le plus grand péril qu'encourent les communautés d'habitants riveraines de la Loire vient du fleuve, qui est sans doute alors une voie majeure de navigation et une artère essentielle pour l'économie de sa vallée mais qui est aussi une source de danger permanent car elle est sujette à de graves crues.

\section{La défense contre les inondations}

La Loire entre en Anjou juste après les confluents avec deux affluents majeurs de rive gauche, I'Indre et la Vienne, qui peuvent lui apporter de gros volumes d'eau et aggraver les effets de ses crues; elle traverse ensuite la province d'est en ouest et elle y reçoit sur sa rive droite comme principal affluent la Maine, qui est formée de la Sarthe, de la Mayenne et du Loir, et qui peut aussi connaître des crues importantes, tout en servant parfois de "déversoir " au fleuve. Les crues et inondations ont été nombreuses au XVIII ${ }^{\mathrm{e}}$ siècle. À Blaison, le procureur fiscal fait dresser un procès-verbal des inondations en 1709 sur ordre du procureur général du parlement de Paris, en 1711 à la demande du seigneur pour faire évaluer les dégâts dans ses propriétés. De plus la configuration du lit du fleuve varie sans cesse : des îlots apparaissent, disparaissent... ; les entreprises des hommes contribuent à les faire s'étendre et à les mettre en valeur ; comme ces îles sont comprises dans la mouvance de la seigneurie ou dans le domaine royal, ces mutations géographiques ne vont pas sans poser de nombreux problèmes juridiques; l'exemple de Chalonnes et de ses nombreuses îles est très éclairant.

66. Dans le duché-pairie de Château-la-Vallière, le juge a eu à traiter une dizaine de cas au XVIII ${ }^{\mathrm{e}}$ siècle. Cf. MAUCLAIR, Fabrice, La Justice au village..., op. cit., p. 184-189.

67. Arch. dép. de Maine-et-Loire, G74. À Chalonnes le 17 mars 1789 le juge reçoit un chirurgien qui a obtenu sa maîtrise en 1779 et a été reçu dans la communauté des chirurgiens d'Angers.

68. Arch. dép. de Maine-et-Loire, 12 B394. 
Pour se défendre contre les effets des crues, dès le Moyen Âge les Angevins riverains ont commencé à construire des petites digues de terre, les "turcies", puis le pouvoir royal a pris en charge l'extension de ce réseau de "levées » et a établi le corps des ingénieurs des turcies et levées; au $\mathrm{XVIII}{ }^{\mathrm{e}}$ siècle leur construction, leur entretien, leur réparation ne relèvent donc pas des seigneurs, à une exception près, celle de l'île de Chalonnes dont le seigneur baron est l'évêque d'Angers qui y perçoit des revenus très importants.

Une requête du procureur fiscal rédigée en $1728^{69}$ rappelle l'origine de ces levées : les habitants des deux paroisses de Chalonnes, ainsi que ceux de Chateaupanne et de Montjean, avaient demandé à l'intendant Hue de Miromesnil l'autorisation de faire renfermer "à leurs frais ce terrain par des turcies et levées, ce qui leur fut octroyé en l'année 1693 et furent construites à frais communs et en conséquence des ordres dudit sieur de Miromesnil il fut fait un rôle par lequel chaque propriétaire d'héritages situés dans ladite isle fut taxé pour contribuer aud. établissement »; ces levées sont donc privées et le juge seigneurial doit en assurer la police. Par la suite, les digues dont l'utilité avait pourtant été reconnue furent plus ou moins bien entretenues et lors des grandes crues elles craquèrent, si bien que les terres furent envahies et recouvertes de sables (par exemple en 1725 et au début 1726, ou dans l'hiver 1727-1728).

Le 21 mai 1720, les propriétaires de l'île de Chalonnes s'assemblent « en conséquence du mandement de Monsieur le sénéchal de cette cour » et se mettent d'accord sur la nécessité des réparations à faire aux levées mais choisissent la voie de l'action individuelle, chacun devant procéder " au rétablissement » des levées bordant ses terres. En mai 1728, parce que les levées avaient cédé en différents endroits lors des crues de l'hiver précédent, parce que les dégâts dus aux crues de 1725 n'étaient pas encore tous réparés, le procureur intervient en urgence et demande " en attendant de se pourvoir devant Mgr l'intendant que les habitants se réunissent pour aviser au meilleur moyen de réparer les levées et prévenir les inondations qu'on a tout lieu de craindre cette année, comme il en arrive presque tous les ans au printemps et lorsqu'on est près de ramasser les ensemencés ». Le sénéchal convoque alors l'assemblée des habitants; une telle procédure avait déjà été utilisée en 1701, du temps de l'intendant Turgot. L'intendant signe, le 29 mai 1728, une ordonnance pour autoriser l'établissement d'un procès-verbal de l'état des levées. Puis, les habitants concernés, refusant que des adjudications au rabais des travaux soient faites, déclarent vouloir réaliser eux-mêmes les réparations; en octobre le procureur fiscal constate que certains n'ont encore rien fait alors que la saison des crues approche et il demande à l'intendant l'autorisation de faire faire les travaux par des ouvriers et des journaliers aux frais des propriétaires négligents; Pomereu

69. Arch. dép. de Maine-et-Loire, 1E178. Une supplique du syndic, en date du 2 novembre 1726, adressée à l'intendant Pomereu, avait déjà porté cette situation à sa connaissance : les propriétaires s'étaient engagés « à l'entretien chacun en droit soy desd. levées ». 
donne son autorisation le 20 octobre suivant. Le sénéchal ordonne alors le " récolement des levées » le 27 octobre; le 30 octobre les deux experts nommés font l'inspection et dressent le procès-verbal de l'état d'avancement des travaux. II semble qu'en 1730 les travaux n'aient guère progressé et que de nouvelles brèches se soient produites, si bien qu'une autre visite est faite et que le même processus est déclenché; c'était encore la répétition de ce qui s'était passé en 1719-1720. Le procureur s'indigne souvent de la passivité des propriétaires de l'île qui ne font rien, alors qu'ils sont intéressés au premier chef. Un processus identique se déroule à plusieurs reprises dans le courant du siècle : le 22 septembre 1751 le procureur fiscal convoque une assemblée générale, non de toute la communauté mais des propriétaires et possesseurs des terres de "la grande île de Chalonnes » et des habitants des lieux, en vertu de l'arrêt du conseil du 21 octobre 1748 et de l'ordonnance de l'intendant du 10 septembre 1751. Elle doit désigner des experts qui feraient le procès-verbal des dégâts causés par les crues de l'hiver précédent; l'assemblée se perd en discussions sur l'opportunité des réparations et ne conclut pas ${ }^{70}$. Les mêmes vains débats se déroulent en janvier 1777. Les officiers seigneuriaux n'ont donc pas pu obtenir l'exécution des ordres : la force d'inertie des habitants l'a emporté et a été plus forte que le sens des réalités.

Les lacunes dans les archives ne permettent que des observations ponctuelles qui peuvent donner une impression de catalogue. Mais tous les silences ne correspondent pas à des absences documentaires. En effet, les officiers seigneuriaux n'interviennent pas dans toutes les affaires de la communauté : ils ne président pas la réunion de l'assemblée générale, ils ne s'occupent pas des affaires de la fabrique et ne s'immiscent pas dans la répartition et la perception des impôts royaux, les interlocuteurs des communautés étant alors les officiers du roi. Les dossiers conservés ont été produits par les justices des seigneuries les plus importantes; il faudrait pouvoir étudier aussi l'activité d'autres justices seigneuriales, plus limitées. Il est donc difficile de savoir si la police était bien exercée dans toutes les seigneuries où juridiquement la possibilité existait, mais aussi de dresser une statistique des affaires de police traitées par les procureurs fiscaux et les juges. Nous n'avons aucune indication sur le degré d'implication des seigneurs dans ce domaine mais il faut se souvenir que l'exercice de ces droits de justice et donc de police avait certainement un coût pour eux. Isoler la police du reste des tâches de la justice stricto sensu amène à oublier certains aspects de la vie sociale : ainsi pour un fonctionnement harmonieux de la société, le "bon ordre des familles » est essentiel et la justice seigneuriale joue un rôle très important dans la vie ordinaire des ménages ruraux. Les attributions en matière de droit familial sont clairement définies et sont bien assumées; elles occupent d'ailleurs une grande partie du

70. Arch. dép. de Maine-et-Loire, 5E53/52, minutes Leduc (acte du 22 septembre 1751) ; 5E53/64, minutes Leduc (acte du 5 janvier 1777). L'arrêt du conseil impose une réunion annuelle pour délibérer « sur la nécessité des réparations à faire à lad. île ». 
temps du juge seigneurial ${ }^{71}$. Cependant il s'agit ici de l'exercice de la justice civile gracieuse, et non de police.

Les observations faites fournissent de nombreuses informations d'une part sur l'évolution des comportements et d'autre part sur les préoccupations principales des officiers de justice, et peut-être des seigneurs. Les juges seigneuriaux ne semblent pas être dans des rapports de concurrence avec les officiers royaux des juridictions ordinaires qui éprouvaient sans doute de la condescendance envers eux. II existe plutôt une répartition des tâches qui est peut-être source de frictions; ainsi, par exemple, les officiers des maîtrises des eaux et forêts ont aussi un rôle important dans la défense des bois ou la réglementation de l'usage des prairies. Il s'est sans doute produit des heurts avec les curés mais ils n'ont, semble-t-il, pas laissé de traces. Très souvent les officiers du seigneur et la communauté d'habitants, qui est moins inculte politiquement au XVIII ${ }^{\mathrm{e}}$ siècle qu'on ne l'a souvent dit, travaillent ensemble : une question est soumise à l'assemblée, elle émet un avis qui est présenté au juge qui le transforme en norme sous la forme d'un règlement et le procès-verbal de l'assemblée devient un titre écrit qui peut entrer dans les archives de la communauté. Dans les moments graves, il s'établit une collaboration entre officiers royaux et seigneuriaux; les procureurs fiscaux sont, avec les curés, les principaux informateurs des autorités régionales ou même nationales : ils écrivent au subdélégué, à l'intendant ou au contrôleur général des finances. Ainsi, le 4 septembre 1771, le procureur fiscal du marquisat de Cholet fait connaître un " enlèvement de grains survenu à Cholet »; le 25 avril 1774, le sénéchal de la terre de Passavant, qui appartient à la marquise de Gouffier, informe le subdélégué de Saumur sur l'état des récoltes et la misère du peuple ${ }^{72}$.

Quelle est l'efficacité réelle de la "police » seigneuriale? Les mesures décidées font-elles un effet durable? On peut en douter : la force d'inertie et la désobéissance sont des armes traditionnelles des populations. Sans doute s'agissait-il d'affaires minimes, triviales, relevant de l'existence quotidienne; mais parce que quotidiennes elles étaient au cœur de la vie des campagnes et la présence d'autorités proches, qui parlaient au nom du roi, était essentielle pour assurer le bon fonctionnement de la vie économique et sociale. En cas de difficultés graves il faut faire appel à la maréchaussée ; toutefois, mis à part les temps de troubles de subsistances, les campagnes angevines apparaissent finalement bien calmes avant $1789 . .$.

En Anjou les officiers seigneuriaux remplissent avec conscience des devoirs qu'ils connaissent bien : la qualité de leur écriture et des textes rédigés prouve qu'ils ont une bonne formation intellectuelle. Les procureurs fiscaux jouent un rôle fondamental dans le fonctionnement du sys-

71. Actes de tutelle et curatelle, entérinement de lettres d'émancipation, apposition de scellés, retraits lignagers, nomination d'experts, etc.

72. Arch. dép. d'Indre-et-Loire, C96 et C97. Les dossiers cotés C94 à C97 contiennent de très nombreuses informations sur l'attitude de plusieurs procureurs fiscaux au cours des les années troublées de 1770 à 1775. 
tème. Comme l'ont constaté différentes études déjà réalisées, qui restent à multiplier, ils ne déméritent en rien. II apparaît clairement que là où ils exercent réellement leurs fonctions en matière de police, les officiers seigneuriaux accomplissent une tâche mutatis mutandis proche de celle des lieutenants généraux de police urbains, et plus généralement ils remplissent, bien qu'appartenant à des "justices subalternes ", les mêmes fonctions que les officiers royaux dont ils se sentent proches.

L'examen de l'exercice de la police dans les campagnes met en évidence une activité qui est le plus souvent négligée dans les études sur la justice considérée trop souvent sous l'angle unique de l'activité au pénal, de la répression des crimes et délits. Juridiquement une justice seigneuriale doit fonctionner dans trois domaines complémentaires : le pénal, le civil non contentieux et la police; en étant efficace dans les trois cas, elle constitue un acteur majeur des régulations sociales dans les campagnes et de la vie politique du royaume.

\section{RÉSUMÉ}

Au XVIII e siècle, en l'absence de tout officier royal de justice et de police dans les campagnes d'Anjou, la police des communautés est assurée par les officiers seigneuriaux de justice des principales terres. Le fonctionnement des ces institutions a produit d'abondantes archives, qui ont été partiellement conservées. En dépit de moyens matériels très limités, ces officiers (juges et procureurs fiscaux) tâchent d'assurer le maintien de l'ordre et de la sécurité, le bon fonctionnement de la vie économique, la police de la vie agraire, la surveillance de la société... Les problèmes à traiter sont le plus souvent sans très grande gravité mais ils sont récurrents. Quand elles sont exercées, ces fonctions de police permettent un encadrement quotidien des communautés rurales et elles ne doivent pas être négligées dans l'étude des justices seigneuriales.

\section{ABSTRACT}

In the $18^{\text {th }}$ century, when no royal law or police officer was to be found in the Anjou countryside, the manorial law officers of the main estates were in charge of community police. Operating those institutions resulted in extensive records, not all of which were destroyed. Despite very limited material means, those officers (judges and fiscal prosecutors) endeavoured to maintain law and order, as well as ensuring a smoothly running economic life, farm life police and the surveillance of society as a whole. More often than not, the problems to be dealt with were rather minor ones, but they occurred regularly. When discharged, these police duties made the daily management of rural communities possible; consequently they should not be ignored when one is studying manorial justice. 
